\title{
EFFECTS OF UNIFIED POWER FLOW CONTROLLER (UPFC) ON DISTANCE RELAY TRIPPING CHARACTERISTICS IN THE NORTH-CENTRAL NIGERIAN 330kV NETWORK
}

\author{
L. Yusuf $1,{ }^{*}$ and M. N. Nwohu ${ }^{2}$ \\ 1, 2 Dept. of Electrical/Electronic Engineering, Federal Univ. of Technology, Minna, Niger State. NiGERIA. \\ Email addresses.1 yusuflatifa@yahoo.com, 2 mnnwohu@futminna.edu.ng
}

\begin{abstract}
This paper investigates the effects of UPFC on Distance Relay tripping characteristics in Nigerian 330kV (NorthCentral) Network. Its operation is based on impedance measurement at the relaying point However, the system performance is often impeded by certain operational or structural factors such as load angle, the voltage magnitude ratio at the line ends, pre-fault line loading and short circuit levels at the line ends. The Unified Power Flow controllers (UPFC) incorporated into the Nigerian $330 \mathrm{kV}$ (North-Central) Network was modelled in the environment of Power System Computer Aided Design (PSCAD) and kept within the protected zone of the relay to increase the Apparent Resistance causing the relay to malfunction. Therefore, it is deduced by simulation analysis that the presence of UPFC in a faulted transmission line loop, protected by distance relay greatly affects the trip boundaries of the distance relay by setting it to either an over reaching or an under reaching state. Hence, the tripping characteristics of distance relay with UPFC located at various points with respect to a fault on a transmission line culminated in three scenarios whose results were presented and discussed in this paper.
\end{abstract}

Keywords: Distance Relay, FACTS, UPFC, PSCAD, Transmission line\& Nigerian Network

\section{INTRODUCTION}

Distance relays are used as main and back-up protection for transmission lines. They have the ability to detect a fault within a pre-set distance along a transmission line or power cable from its location [1].Its operation is based and governed by the ratio of applied voltage to current, which can be affected by several factors, including pre-fault line loading and short circuit levels at the line ends [2]. The basic principle of distance protection involves the division of voltage at the relaying point by the measured current. The calculated apparent impedance is compared with the reach point impedance. If the measured impedance is less than the reach point impedance, it is assumed that a fault exists on the line between the relay and the reach point. Distance relay is designed to operate only for faults occurring between the relay location and the predetermined (reach) point [1]. However, in the presence of Flexible Alternating Current Transmission System (FACTS) devices such as UPFC in particular, the conventional distance characteristic are greatly subjected to malfunction in the form of over-reaching or underreaching the fault point [3]. The control characteristics of the UPFC, the fault location with respect to the UPFC position greatly affects the trip boundaries of the distance relay on the transmission line and also very high fault resistance makes this problem more severe and complicated [4].

The issue of increasing loads at limited paths for new transmission lines has been reduced by introduction of FACTS devices into the network. Increased interests in improving the transmission system capability and power system controllability have resulted into widespread application of the FACTS [5]. The advent of FACTS controllers in the power system transmission open up new challenges to the line protection as they change the impedance of the lines dynamically. This is because of the added complexity due to the interaction of FACTS devices with the transmission system. The transients superimposed on the power frequency, voltage and current waveforms, particularly, at the occurrence of fault significantly differ from those systems not connected with FACTS 
devices. Consequently, this phenomenon results in rapid changes in system parameters like line impedance and power angle.

\section{DISTANCE RELAY AND ITS TRIPPING CHARACTERISTICS}

Distance Relay is used as main or backup protection for transmission lines. Its principle is based on the division of the voltage at the relaying point by the measured current. The operating characteristics of many distance relays can be expressed in terms of the impedance or its components when plotted on a set of rectangular coordinates ( $\mathrm{R}$ and $\mathrm{X}$ ) whose characteristics form geometrical figures. The operating characteristic of a mho distance relay, also known as an admittance relay, is a circle that passes through the origin of the R-X plane. The third quadrant of the R-X plane is outside the operating characteristic of the relay, hence the faults on the bus side are not seen by the relay. The operating characteristics of a mho Relay is shown in Figure 1 [11].

Distance relay performance is defined in terms of reach accuracy and operating time. Reach accuracy is a comparison of the actual ohmic reach of the relay under practical conditions with the relay setting value in ohms [7]. Distance relays will have instantaneous directional zone 1 protection and one or more time delayed zones. The tripping signal produced by zone 1 is instantaneous which is set to cover only 80-85 per cent of the protected line. The remaining 20-15 percent provides a factor of safety in order to mitigate against errors introduced by the current and voltage transformers, and line impedance calculations. The 20-15 percent at the end of the line is protected by zone 2, which operates in 2 seconds. Zone 3 provides the back-up and operates with a time delay of 3 seconds [8]. The basic protection scheme is shown in Figure 2 [11].

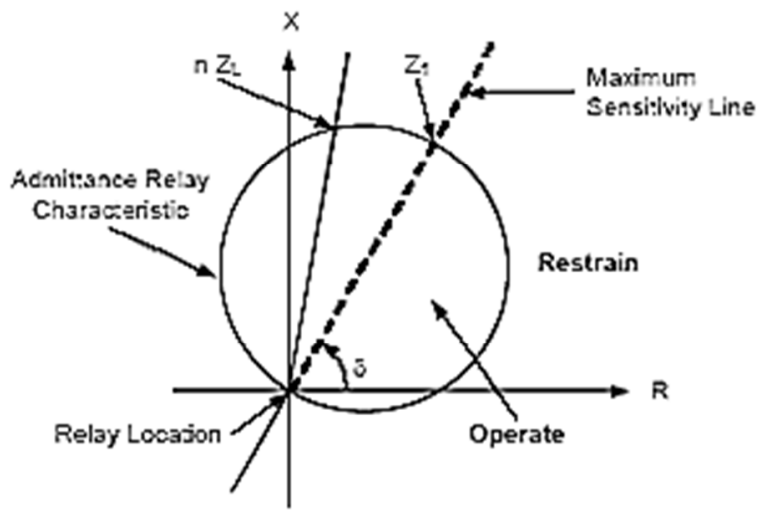

Figure 1: Operating characteristics of mho (admittance relay)

\section{ANALYSIS OF APPARENT IMPEDANCE}

This section presents the mathematical analysis of the apparent impedance seen by the relay. The Apparent Impedance calculation is based on symmetrical component transformation using power frequency amplitude of voltage and current signal measured at relay point. For purpose of analysis, two hypothetical places are faulted $F_{A}$ and $F_{B}$ (see Figure 3 ) which are:

a Fault between relay and UPFC: When fault occurs before UPFC, the Apparent Impedance of distance relay can be calculated using the conventional equations [7].

b. The UPFC between relay and fault: In this situation, the UPFC is at the fault path, the Apparent Impedance seen by the relay for phase to ground fault and phase to phase faults are shown below [9].

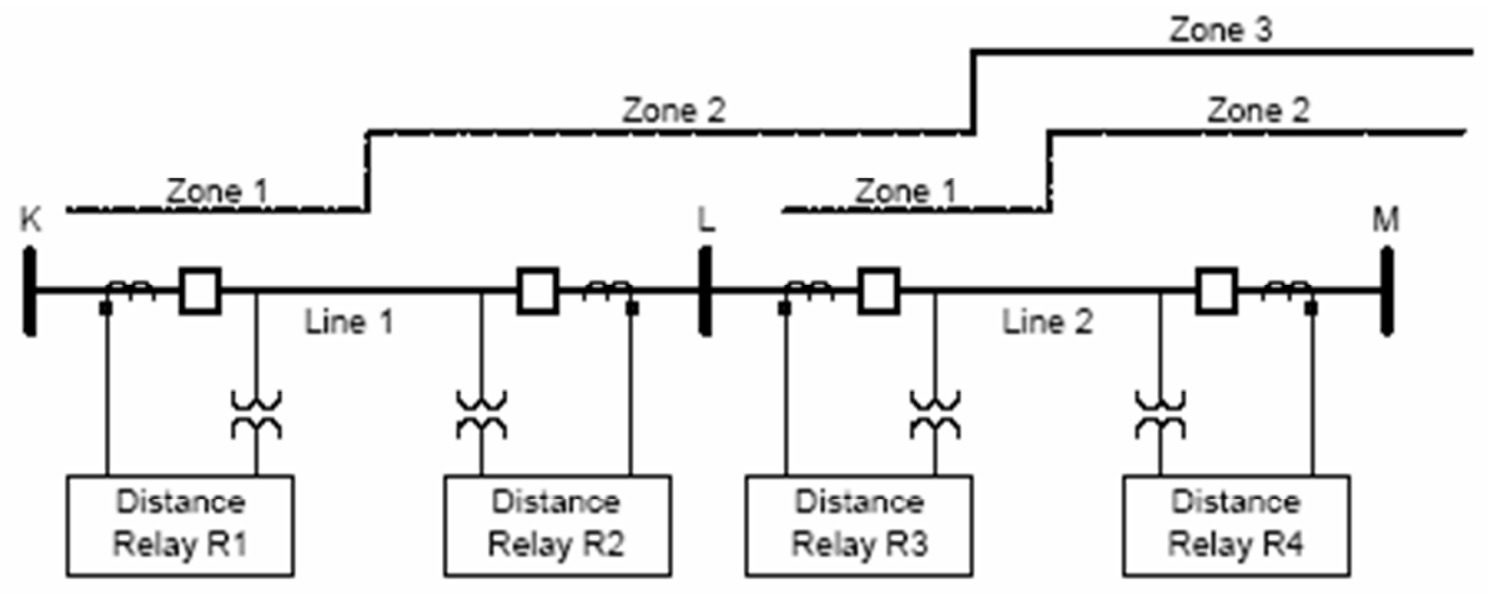

Figure 2: Basic Distance protection scheme 


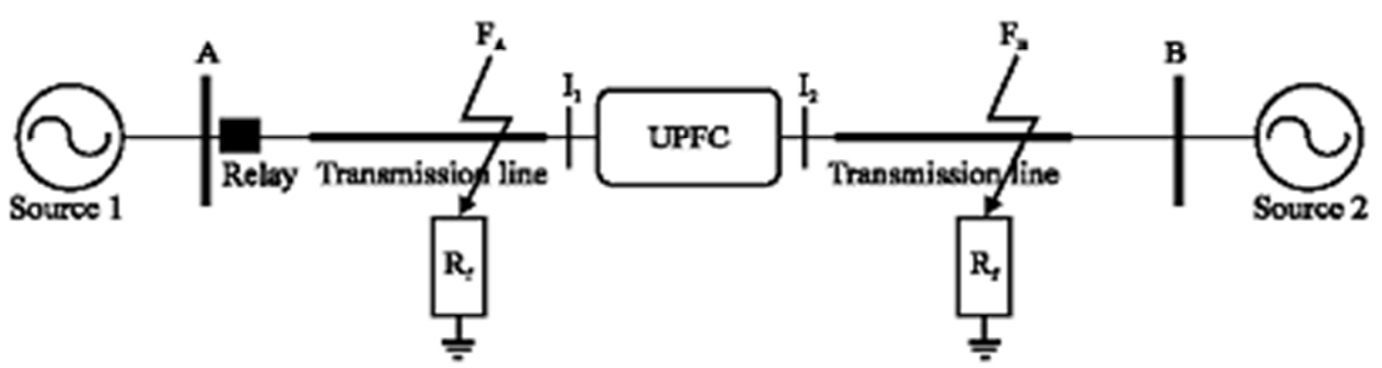

Figure 3 : Hypothetical network for Apparent Impedance Analysis

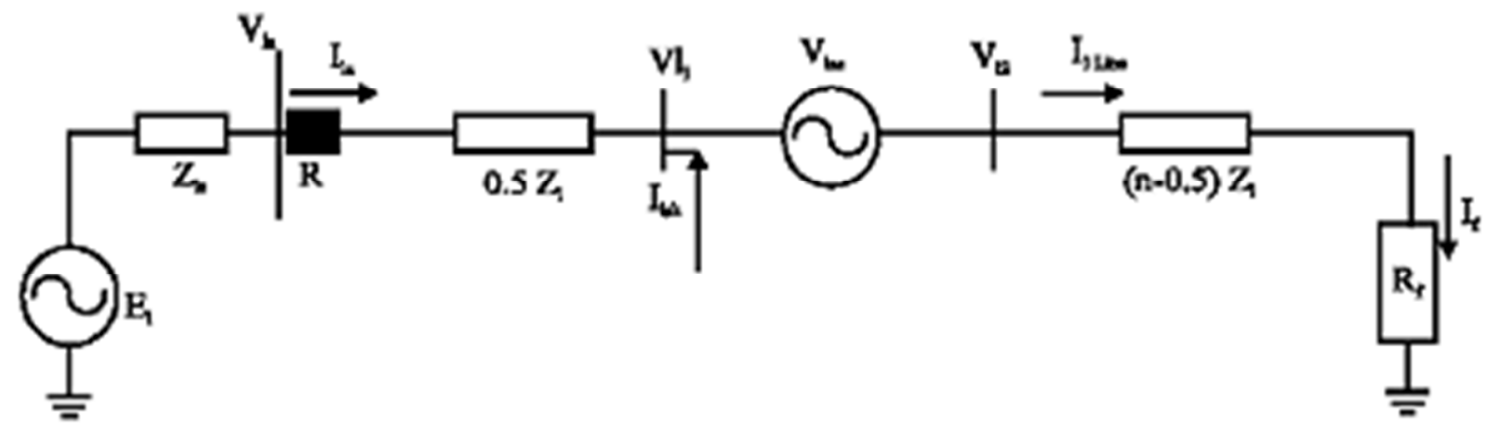

Figure 4: The positive system network of the system from relay point to UPFC

From Figure 4,

$$
\begin{gathered}
V_{1 A}=I_{1 A} 0.5 Z_{1}+V_{1 S E}+I_{1 \text { Line }}(n-0.5) Z_{1} \\
+R_{f} I_{1 f} \\
V_{2 A}=I_{2 A} 0.5 Z_{1}+V_{2 S E}+I_{12 \text { Line }}(n-0.5) Z_{1} \\
+R_{f} I_{2 f} \\
V_{0 A}=I_{0 A} 0.5 Z_{1}+V_{0 S E}+I_{0 \text { Line }}(n-0.5) Z_{1} \\
+R_{f} I_{0 f} \\
I_{1 \text { Line }}=I_{1 A}+I_{1 s h} \\
I_{2 \text { Line }}=I_{2 A}+I_{2 s h} \\
I_{0 \text { Line }}=I_{0 A}+I_{0 s h}
\end{gathered}
$$

where: $V_{1 A}, V_{2 A}, V_{0 A}$ are the sequence phase voltages at relay location, $V_{1 S E}, V_{2 S E}, V_{0 S E}$ are the series sequence phase voltages injected by UPFC; $I_{1 A}, I_{2 A}, I_{0 A}$ are the sequence phase currents at relay location; $I_{1 \text { Line }}, I_{2 \text { Line }}, I_{0 \text { Line }}$ are the sequence phase currents in the line. $\left(Z_{2}=Z_{1}\right) ; \quad I_{1 f}, I_{2 f}, I_{0 f}$ are the sequence phase currents of the fault; $I_{1 s h}, I_{2 s h}, I_{0 s h}$ are the shunt sequence phase currents injected by UPFC; $Z_{1}, Z_{0}$ are the sequence impedances of transmission line; $E_{1}$ is the positive sequence of voltage source. $Z_{1 s}, Z_{2 s}, Z_{0 s}$ are the sequence of the source impedances, $n$ is the per unit distance of a fault from the relay location. $L$ is the length of the transmission line $n \times L$ is the fault location.

The positive, negative and zero sequence circuit diagram for Figure 3 is shown in Figure 5.

The measured impedance at relaying point for a phase to ground fault at the transmission line can be calculated by the following equations

$$
Z_{R}=\frac{V_{A}}{I_{A}-\frac{Z_{0}-Z_{1}}{Z_{0}} I_{A 0}}=\frac{V_{A}}{I_{\text {relay }}}
$$

where $V_{A}$ is the voltage at the relay point. From Equations 1 to 3, it can be derived as:

$$
\begin{aligned}
V_{A}=n I_{a}+ & n I_{0 a}\left(Z_{0}-Z_{1}\right)+I_{s h}(n-0.5) Z_{1} \\
& +(n-0.5) I_{0 s h}\left(Z_{0}-Z_{1}\right)+V_{S E} \\
& +R_{f} I_{f}
\end{aligned}
$$

Where:

$$
\begin{gathered}
V_{A}=V_{1 A}+V_{2 A}+V_{0 A} \\
I_{a}=I_{1 a}+I_{2 a}+I_{0 a} \\
I_{S h}=I_{1 s h}+I_{2 S h}+I_{0 S h} \\
V=V_{1 S E}+V_{2 S E}+V_{0 S E}
\end{gathered}
$$




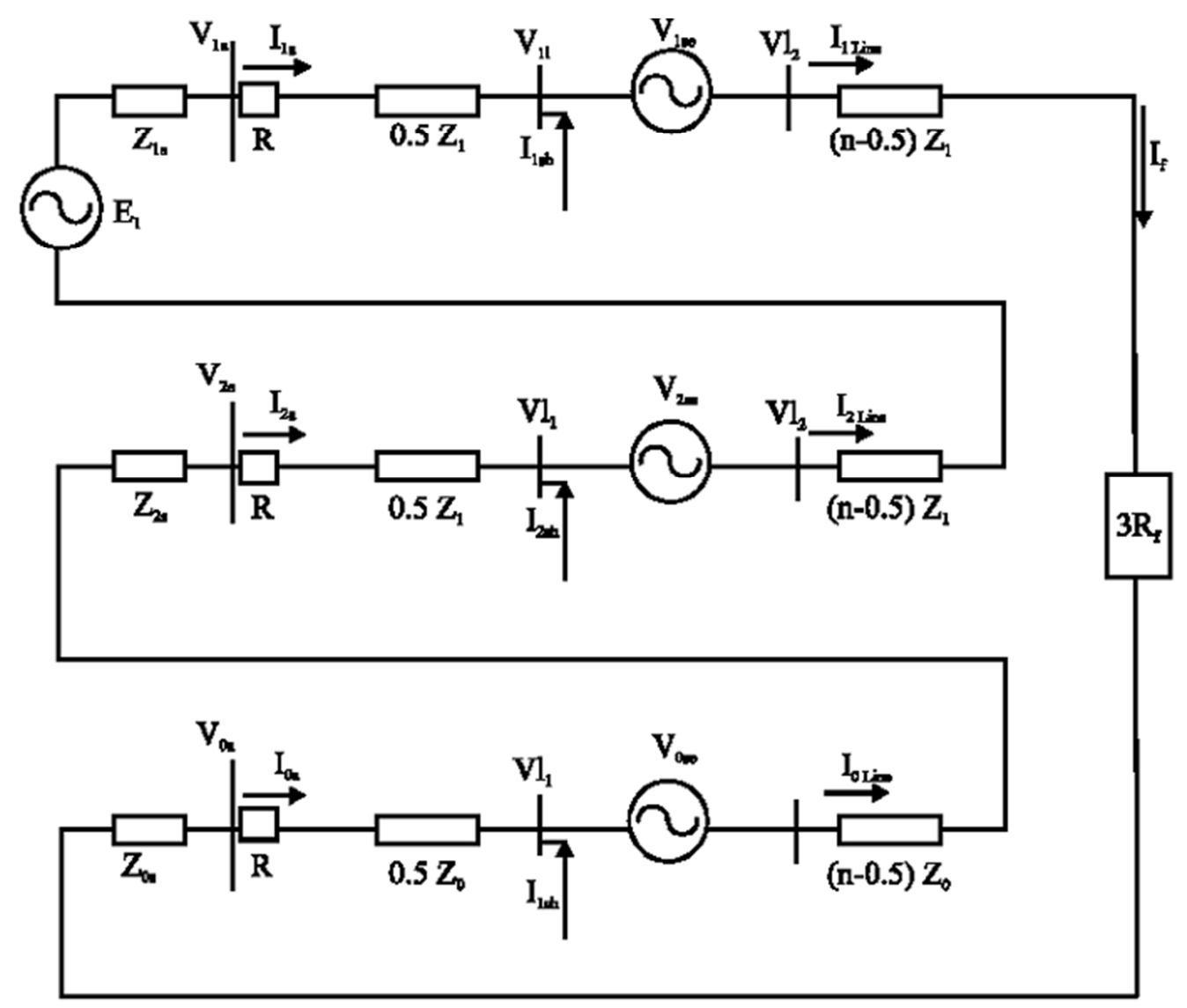

Figure 5: Sequence diagram for single phase to ground fault.

By substituting Equation (8) into Equation (7), the Apparent Impedance can be obtained thus,

$$
Z_{R}=n Z_{1}+\frac{I_{s h}}{I_{\text {relay }}}(n-0.5) Z_{1}+\frac{I_{0 s h}}{I_{\text {relay }}}(n-0.5)\left(Z_{0}-Z_{1}\right)+\frac{V_{S E}}{I_{\text {relay }}}+\frac{I_{f}}{I_{\text {relay }}} R_{f}
$$

In Equation 13, $I_{0 s h}$ is assumed zero because in practice, one side of the shunt transformer is often based on delta connection.

The measured Impedance at the relaying point for phase to phase fault at the transmission line can be calculated by the following equations:

$$
Z_{R}=\frac{V_{a 1}-V_{a 2}}{I_{a 1}-I_{a 2}}=\frac{V_{b}-V_{c}}{I_{b}-I_{c}}
$$

where $V_{b}, V_{c}$ are phase voltages; $I_{b}, I_{C}$ are phase currents and $Z_{R}$ is the measured Impedance by the relay.

NB: For phase-to-phase fault there is no zero sequence networks involved.

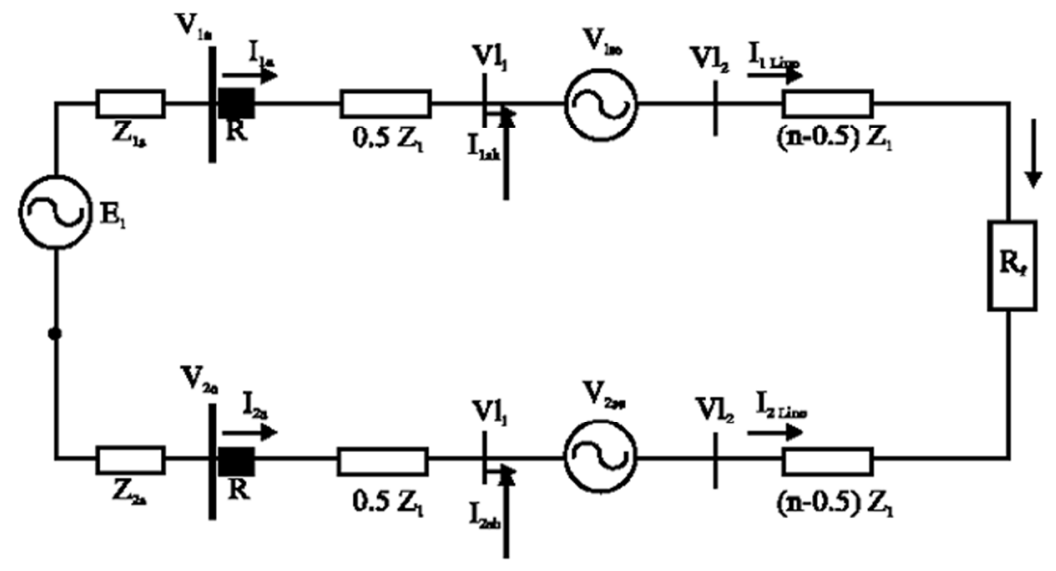

Figure 6: Sequence circuits for a phase to phase fault 


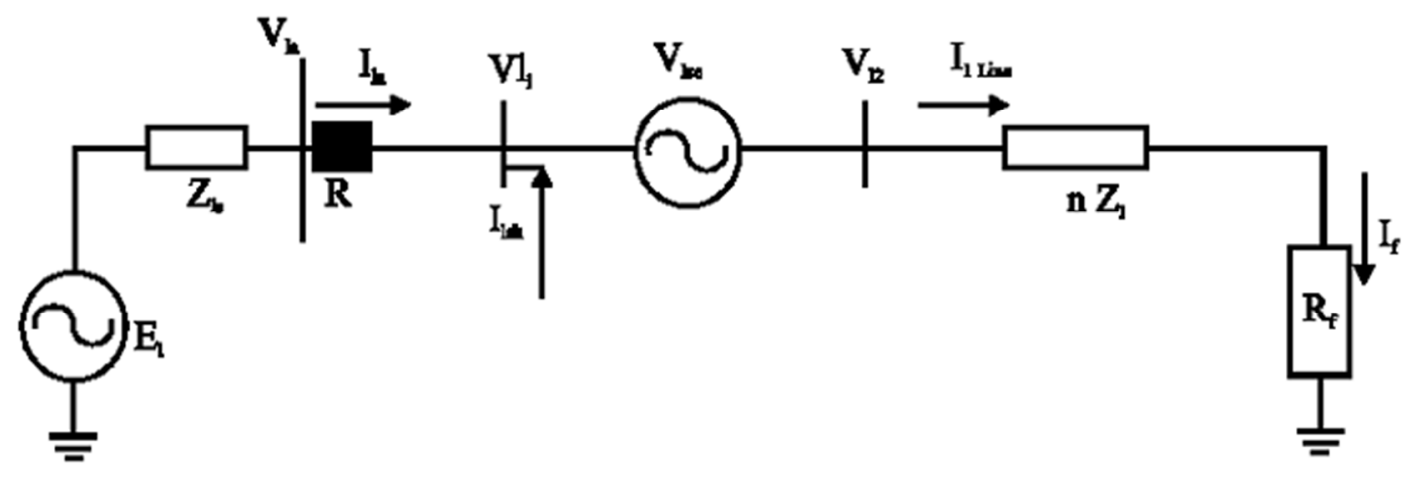

Figure 7: Positive sequence of the network from relay point to fault (with UPFC at the beginning of the line)

If the phase to phase fault in the presence of UPFC at the midpoint of transmission line occurs by substituting equation of the Apparent Impedance by the relay will be derived as

$$
Z_{R}=n Z_{1}+\frac{I_{b s h}-I_{c s h}}{I_{b}-I_{c}}(n-0.5) Z_{1}+\frac{V_{b s e}-V_{c s e}}{I_{b}-I_{c}}+\frac{I_{b f}-I_{c f}}{I_{b}-I_{c}} R_{f}
$$

From equations (13) and (15), it can be seen that UPFC would affect the measured impedance at the relay by two terms, one results from the shunt current by the STATCOM and the other is as result of the series voltage by SSSC, which are components of UPFC.

In Figure 7 when UPFC is installed at the beginning of the line, the measured impedance in the case of phase to ground fault is gotten by modifying equations (13) and (14). The resulting equation is:

$$
Z_{R}=n Z_{1}+\frac{I_{\text {sh }}}{I_{\text {relay }}} n Z_{1}+\frac{I_{0 s h}}{I_{\text {relay }}} n\left(Z_{0}-Z_{1}\right)+\frac{V_{\text {se }}}{I_{\text {relay }}}+\frac{I_{f}}{I_{\text {relay }}} R_{f}
$$

For phase to phase we have,

$$
Z_{R}=n Z_{1}+\frac{I_{b s h}-I_{c s h}}{I_{b}-I_{c}} n Z_{1}+\frac{V_{b s e}-V_{c s e}}{I_{b}-I_{c}}+\frac{I_{b f}-I_{c f}}{I_{b}-I_{c}} R_{f}
$$

\section{DYNAMIC MODELLING OF UPFC}

Figure 8 represents a one-line circuit diagram model of UPFC installed in a power system equipped with a UPFC. The series and shunt Voltage Sourced Inverters (VSIs) are represented by controllable voltage sources $V_{c}$ and $V_{p}$, respectively. $R_{p}$ and $L_{p}$ represent the resistance and leakage reactance of the shunt transformer [10].

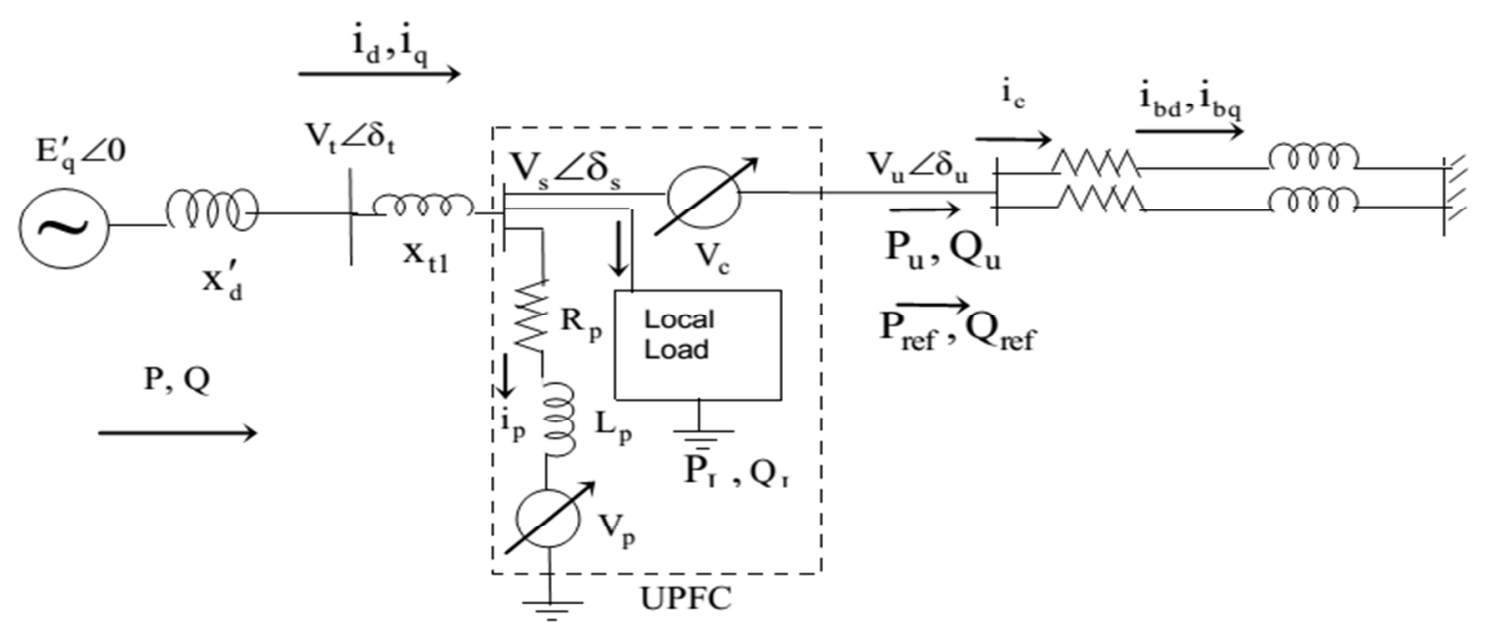

Figure 8: One-line circuit diagram model of UPFC installed in a power system

The dynamic model of UPFC is derived by performing standard d-q transformation of the current through the shunt transformer and series transformer and is presented thus;

$$
\frac{d i_{p d}}{d t}=-\frac{R_{p}}{L_{p}} i_{p d}+w i_{p q}+\frac{1}{L_{p}}\left(V_{s d}-V_{p d}\right)
$$




$$
\begin{aligned}
& \frac{d i_{p q}}{d t}=-\frac{R_{p}}{L_{p}} i_{p q}+w i_{p d}+\frac{1}{L_{p}}\left(V_{s q}-V_{p q}\right) \\
& \frac{d i_{b d}}{d t}=-\frac{w_{b} r_{e}}{x_{e}} i_{b d}+w i_{b q}+\frac{W_{b}}{X_{e}}\left(V_{u d}-V_{b} \sin \delta\right) \\
& \frac{d i_{b q}}{d t}=-\frac{w_{b} r_{e}}{x_{e}} i_{b q}+w i_{b d}+\frac{W_{b}}{X_{e}}\left(V_{u q}-V_{b} \cos \delta\right)
\end{aligned}
$$

where $\omega$ is the angular frequency of the voltages and currents.

For fast voltage control, the net input power should instantaneously meet the charging rate of the capacitor energy. Thus, by applying power balance conditions, we obtain the equations;

$$
\begin{array}{r}
P_{s}-P_{u}=V_{s d}\left(i_{p d}+i_{b d}\right)+V_{s q}\left(i_{p q}+i_{b q}\right)-\left(V_{u a} i_{b d}+V_{u d} i_{b q}\right) \\
V_{d c}=\left[C \frac{d V_{d c}}{d_{t}}+g_{c p} V_{d c c}\right] \\
\frac{d V_{d c}}{d t}=-\frac{g_{c p} w}{b_{c p}} V_{d c}+\frac{1}{C V_{d c}}\left[V_{s a} i_{p d}+V_{s q} i_{p q}+\left(V_{s d}-V_{u d}\right) i_{b d}+\left(V_{s q}-V_{u q}\right) i_{b q}\right]
\end{array}
$$

\section{SIMULATIONS}

The Nigerian 330kV grid system of North-Central Region (which comprises of Birnin Kebbi, Kaduna, Jebba, Kainji and Shiroro), UPFC and distance relay (mho type) was modelled and simulated in Power System Computer Aided Electromagnetic Transient \& DC (PSCAD/EMTDC) environment as depicted in Figure 10. The successful implementation of UPFC on Nigerian power network could be attributed to the dynamics of its controls as shown in Figure 9.

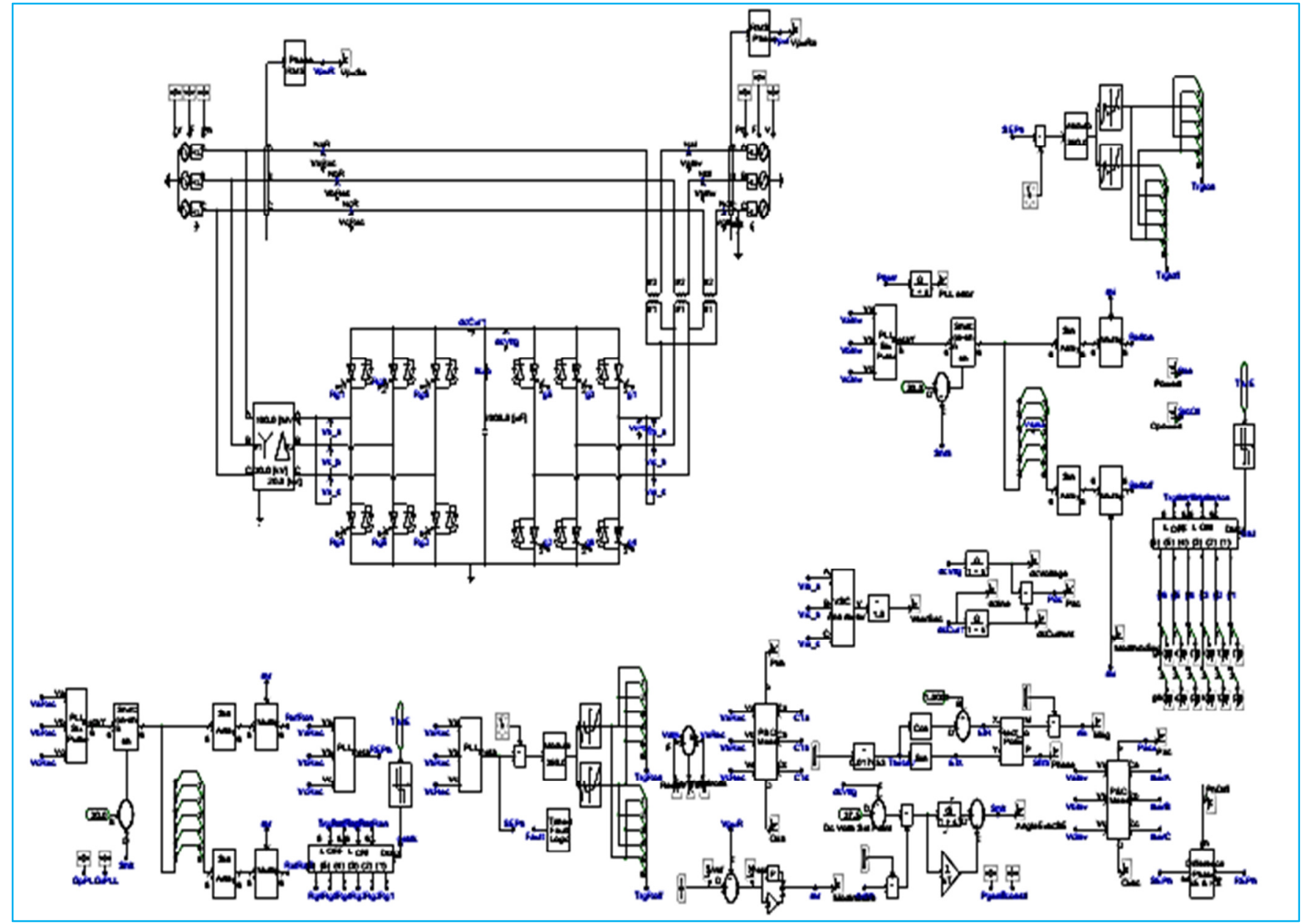

Figure 9: PSCAD Implementation of UPFC 


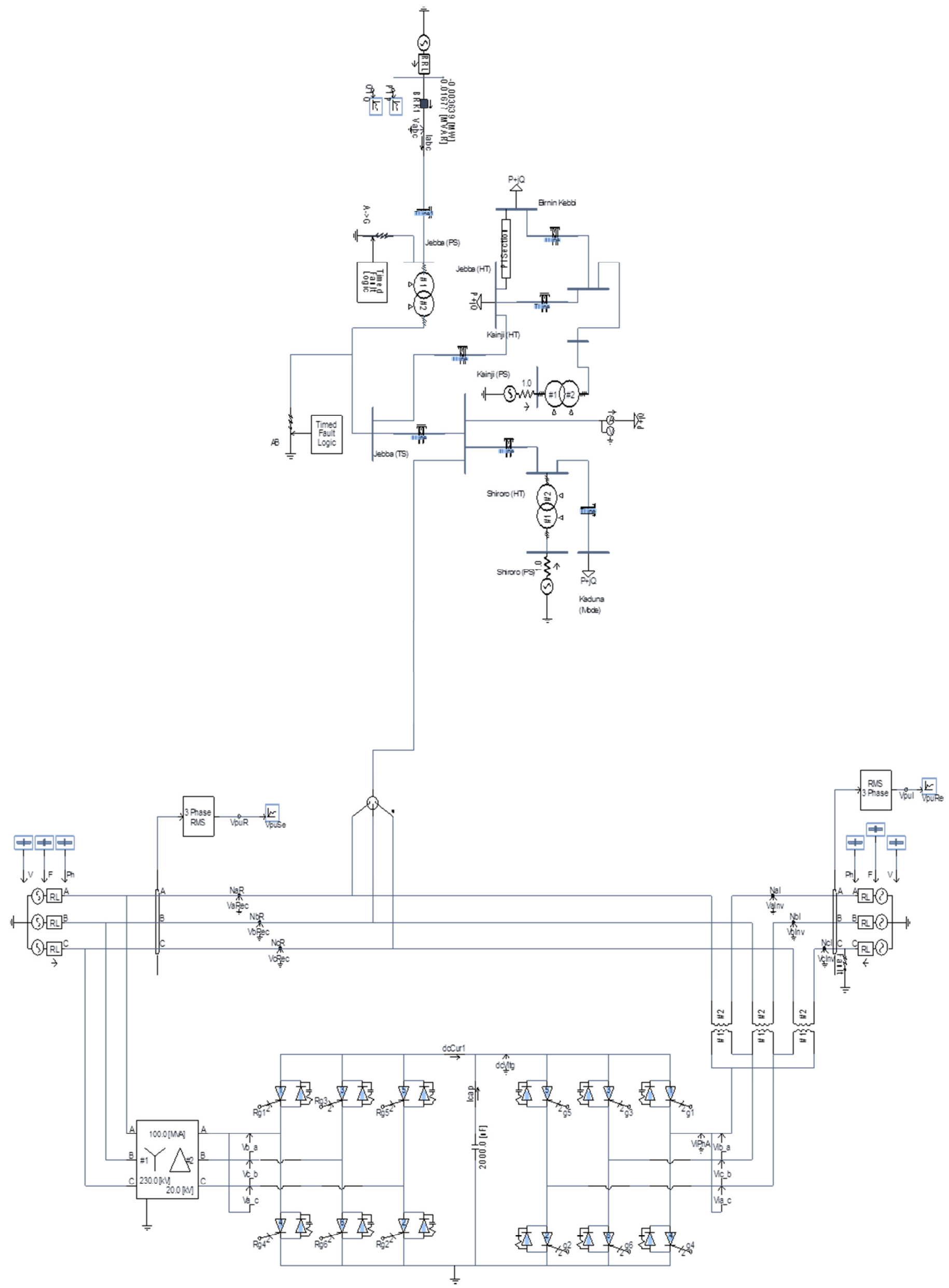

Figure 10: Nigerian (North-Central) 330kV Grid System with Relay and UPFC 


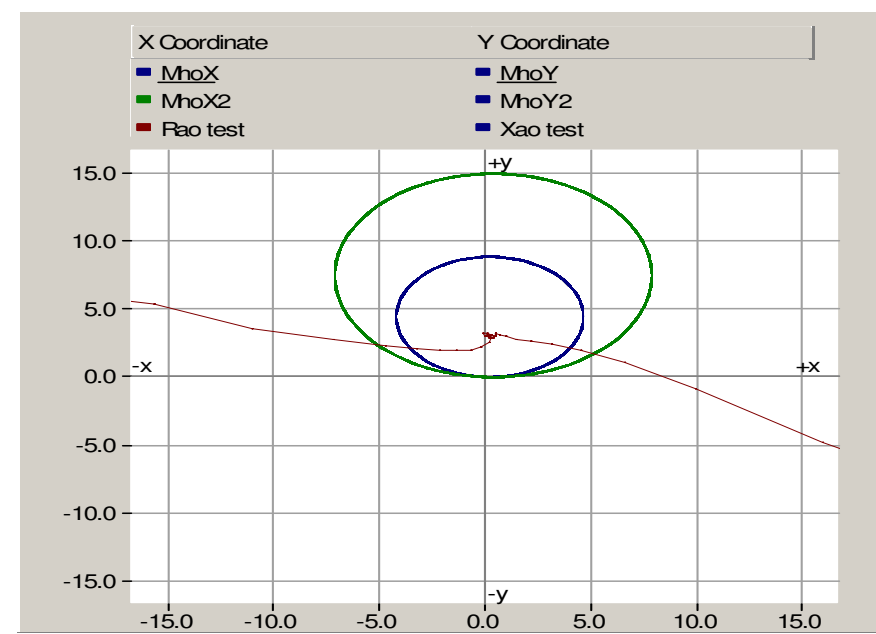

Figure 11: Representation of the fault zones by the distance relay with UPFC placed before a fault.

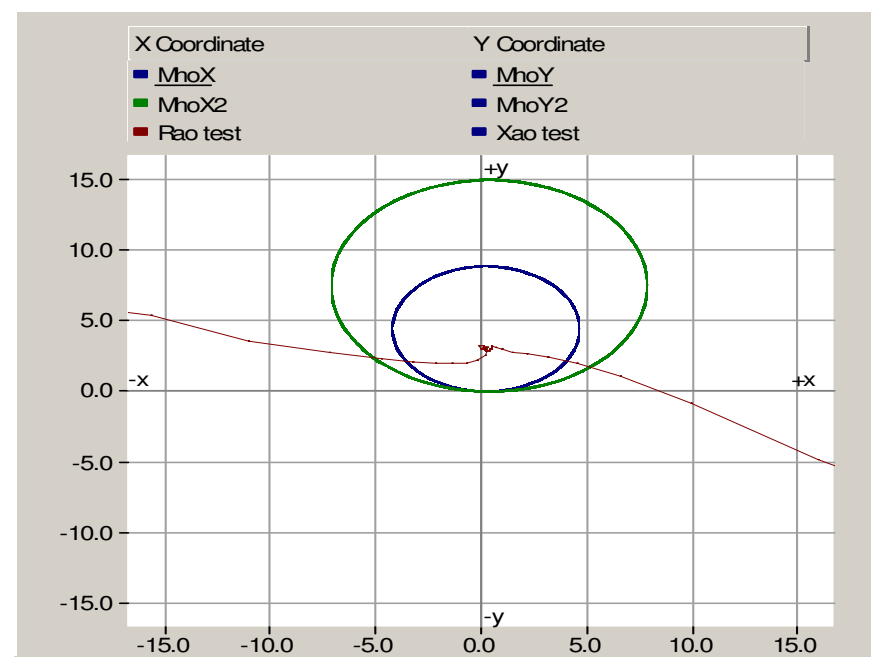

Figure 13: Representation of the fault zones by the distance relay without UPFC

The protection system consists of two operating relaying zones, namely, zone-1 that performs primary protection and zone-2 that performs back-up, or remote-trip, protection. The transmission line length was set to be $125 \mathrm{~km}$, frequency of $50 \mathrm{~Hz}$, and fault on resistance to be $0.1 \Omega$. A three phase fault was initiated on the line at $50 \mathrm{~km}$ of the line. This implies that the zone 1 distance of the relay is $100 \mathrm{~km}$ ( $80 \%$ of the protected line). The setting value in terms of the desired voltage for UPFC is 1.0pu. The UPFC was placed at various points $(30 \mathrm{~km}$ before fault, $20 \mathrm{~km}$ after fault point and placed on another bus in the network). Then the network was simulated without UPFC to see the effect on the trip boundary of the relay. The three phase fault was set to occur at $0.491 \mathrm{sec}$ at the start of the simulation.

In order to investigate the behaviour of the distance relay tripping boundaries upon application of UPFC, three scenarios were studied:

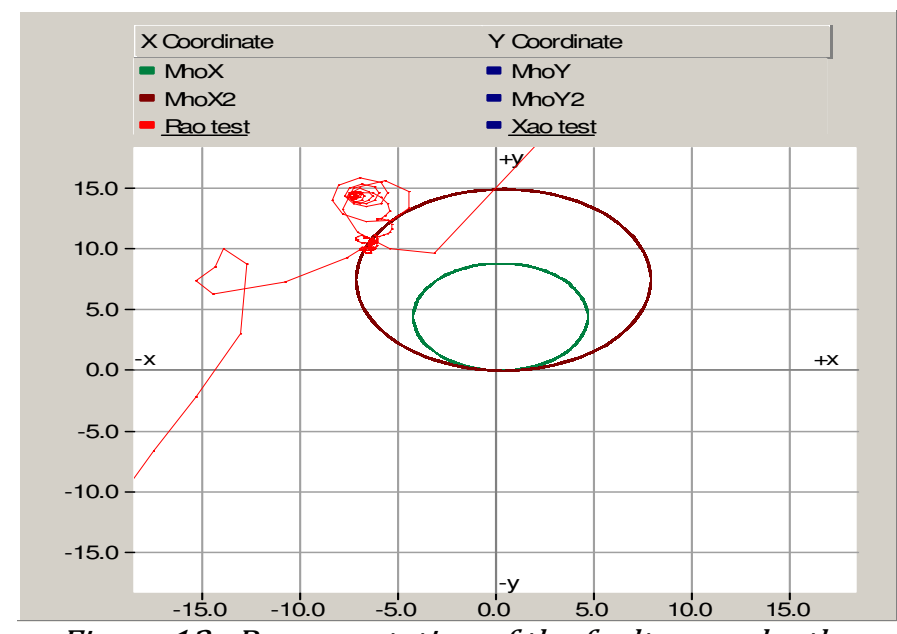

Figure 12: Representation of the fault zones by the distance relay with UPFC placed after a fault

- UPFC placed before the fault loop, UPFC placed after the fault loop and The network without UPFC

\section{RESULTS AND DISCUSSIONS 6.1 UPFC placed before fault}

From Figure 11, the blue circle (smaller circle) represents Zone 1 while the green circle represents Zone 2 (bigger circle) of the relay. The UPFC was placed at $30 \mathrm{~km}$ of the transmission line which is exactly $20 \mathrm{~km}$ before the fault. For the fault initiated, the relay tripped in zone 1 , which is a correct operation of the relay. The relay tripped without having any interference from the UPFC, as the UPFC is not within the fault loop.

\subsection{UPFC placed after fault}

In Figure 12, the green circle (smaller circle) represents zone 1 while the brown circle (bigger circle) represents zone 2 of the relay. The UPFC was placed at $70 \mathrm{~km}$ of the transmission line which is $20 \mathrm{~km}$ after the fault. Now, the relay tripped in zone 2, which is a malfunction of the relay (under reach) because at that distance, the relay is supposed to see the fault in zone 1 . This malfunction can be attributed to the influence ratio $\left(I_{\text {sh }} / I_{\text {relay }}\right)$. Influence ratio becomes higher because of reactive power injection by STATCOM.

\subsection{The Network without UPFC}

In Figure 13, the blue circle (smaller circle) represents zone 1 while the green circle (bigger circle) represents zone 2 of the relay. The UPFC was totally removed from the network and the fault was 
introduced at $50 \mathrm{~km}$ of the transmission line. The relay tripped in zone 1 , (because the fault is within Zone 1 of the relay), which is a normal operation.

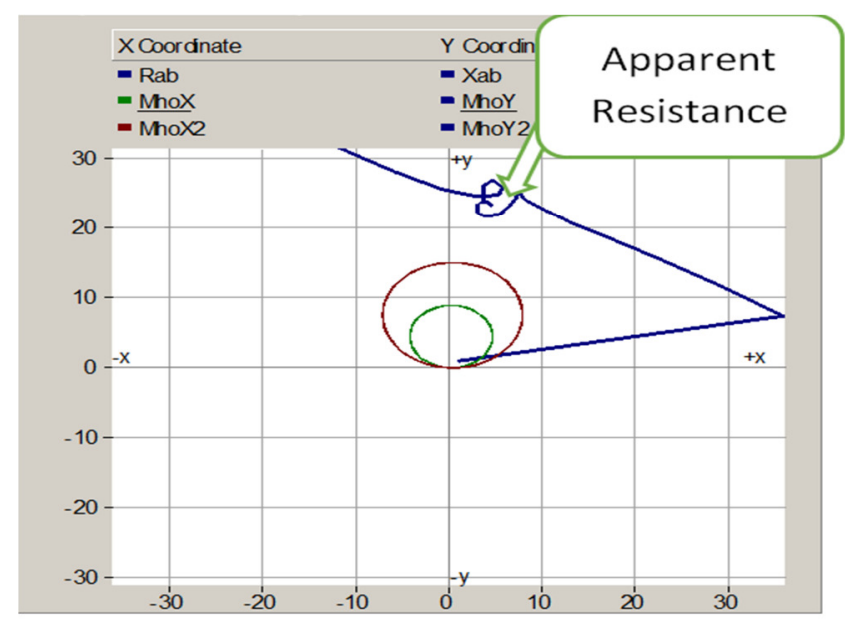

Figure 14: Apparent Resistance with UPFC before the fault loop

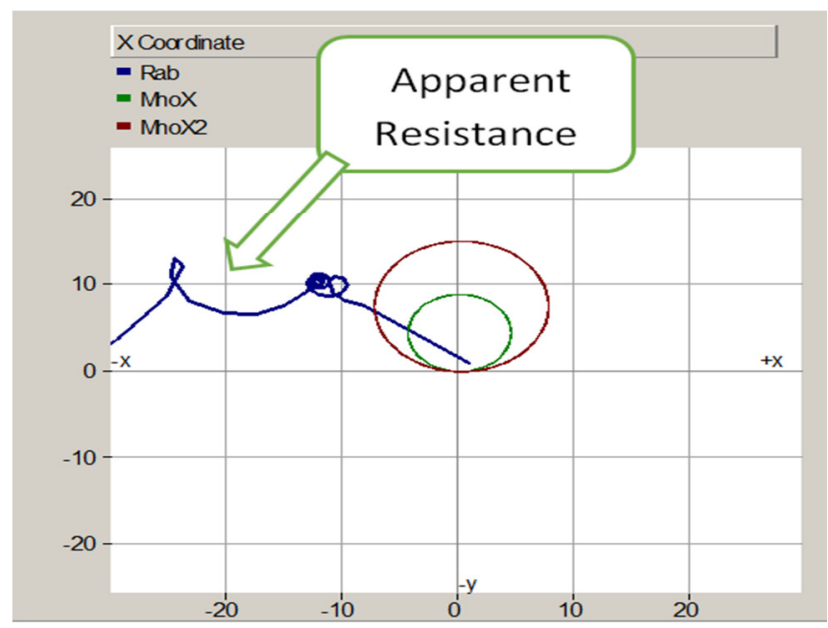

Figure 15: Apparent Resistance with UPFC in the fault loop

\subsection{Comparison of the Apparent Resistance seen by the Relay}

From the Simulation results, the mal function of the relay (with UPFC placed after fault) was as a result of the sharp increase of the Apparent Resistance seen by the relay through the UPFC. This made the relay to under reach, thereby tripping in zone 2 instead of zone1. The deviation in Apparent Resistance is shown in Figures 14 and 15.

\section{CONCLUSION}

It has been established in this paper that the presence of UPFC in a faulted Nigerian (North-Central) $330 \mathrm{kV}$ Network considered and protected by distance relay greatly affect the trip boundaries of the distance relay by setting it to either an over reaching or an under reaching state. UPFC presented within a fault loop, thwarted the normal operation of a distance relay. This is because of the distortion of Apparent Resistance caused by UPFC that was noticed by the distance relay. Of course, this effect is highly undesirable considering the very critical operations of distance relays. Finally, it was observed that the absence of UPFC in the network and faulted loop does not affect the functionality of the relay, hence if UPFC is present in a network in the presence of distance relay, an Adaptive Relay setting is required to overcome the UPFC effect

\section{REFERENCES}

[1] T.A Yumak,Distribution of Electrical Energy Lab. Notes v1.0".http://web.itu.edu.tr/ yumakk/ downloads/Lab Notes Distance Protection v1.pdf, Accessed on March 102015 .

[2] S.Jamali, A.Kazemi, and H.Shateri, "Distance Relay Tripping Characteristics in Presence of UPFC",Power Electronics, Drives and Energy Systems, pp. 1-6, (2006)

[3] A.Abdolkhani, S.P.Bandaghiri, and F.Rocky "Impact of STATCOM on Distance Relay". International Journal of Advanced Research in Electrical Electronics and Instrumentation Engineering, vol 2(issue 10), pp 1-5, , (2013).

[4] P.K.Dash, A.K. Pradhan, G.Panda, and A. C. Liew "Adaptive Relay Setting for flexible AC transmission systems (FACTS)". IEEE Transaction on Power Delivery, vol.15, pp. 38-43, (2000).

[5] N.G Hingorani and L.Gyugyi,"Understanding Facts, Concept and Technology of AC Transmission System". IEEE Transaction on Power Delivery, pp 15, (2000).

[6] J.J.Paserba, "How FACTS Controllers Benefits AC Transmission System", IEEE Power Engineering, (2004), pp 1-8

[7] G.Ziegler "Numeric Distance Protection, Principles and Application". Wiley and sons, Berlin Germany, 2006.

[8 El Arroudi, K.G. Joos and McGillis "Operation of Impedance Protection relay with the STATCOM". IEEE Transaction on Power Delivery, pp. 381-387, (2002)

[9] X.H. Wang, R. K. Aggarwal and P. Beamount "Performance Evaluation of Distance Relay applied to a Transmission system with UPFC" IEEE Transaction on Power Delivery, vol 15, pp 11371147, (2006)

[10] P.Kumar, K. Sahoo, and P. K. Hota "Modeling and simulation of UPFC using PSCAD/EMTDC" International Journal of Physical Sciences, vol 7, issue 45, pp.5965-5980, (2012)

[11] Extract from CIGRE Publications Working group, http://pangolino.com/?sdmon=files, Accessed on March 152015. 